\title{
Der Whistleblower im Spannungsfeld zwischen Korruptionsbekämpfung und arbeitsrechtlichen Pflichten
}

\section{Einfübrung}

Korruptionsbekämpfung in Wirtschaft und öffentlicher Verwaltung ist heute in aller Munde. So hat etwa am I I. Juli 2006 der Europäische Gerichtshof festgestellt, dass Edith Cresson ihre Pflicht als Europäische Kommissarin mit der Einstellung eines persönlichen Beraters als Gastwissenschaftler aus Art. 2 I 3 Abs. 2 EG verletzt hat. ${ }^{I}$ Laut einer Pressemeldung im Mai 2007 sei einem deutschen Konzern infolge einer Schmiergeldaffäre die »komplette Führung abhanden gekommen «. ${ }^{2}$ Die Bundesregierung arbeitet gegenwärtig an einem Entwurf zu einem Zweiten Korruptionsbekämpfungsgesetz. Dadurch soll die Strafbarkeit von Korruption in Bezug auf deutsche und ausländische Amtsträger harmonisiert werden. Im privatwirtschaftlichen Bereich soll die Strafbarkeit der Angestelltenbestechung gemäß \299 StGB ausgeweitet werden, sodass jede Bestechungshandlung, auch die außerhalb konkreter Wettbewerbssituationen, erfasst würde. ${ }^{3}$ Ist zwar der Ruf nach Korruptionsbekämpfung überall zu hören, darf dennoch der sehr viel weitreichendere Rahmen darüber hinausgehender Delikte nicht vergessen werden. So weisen etwa Peemöller/Hofmann auf eine lange Liste nationaler und internationaler »Bilanzskandale « hin. ${ }^{4}$ Unter dem Titel »Bilanzskandale, Delikte und Gegenmaßnahmen « beschreiben sie eine Wirtschaftsgeschichte der besonderen Art. Doch gleich, um welche Straftaten es sich handelt, es sind am Ende oft Whistleblower oder sogenannte Hinweisgeber, 5 die schwerwiegende Rechtsverstöße aus dem Handeln von Unternehmen, sogenannte Fälle von Wirtschaftskriminalität, offenbaren. ${ }^{6}$ Straftaten oder auch Ordnungswidrigkeiten, wie beispielsweise kartellrechtliche Verstöße, gelangen so zur Anzeige. ${ }^{7}$ Aus kriminalpolitischer Sicht gibt es ein hohes Interesse an derartigen Offenbarungen, sei es auch nur durch eine anonyme Anzeige. ${ }^{8}$ Zahlreiche im Zusammenhang mit unternehmerischem Handeln stehende Straftatbestände 9 sind nicht auf den Schutz individueller, sondern allgemeiner Rechtsgüter gerichtet. ${ }^{I \circ}$ Beispielhaft sei nur auf die $\int \mathbb{S} 264,{ }^{\mathrm{II}}$

I EuGH, Urteil vom i I.07.2006, C-432/04.

2 Frankfurter Allgemeine Sonntagszeitung vom 20. Mai 2007, S. 27.

3 Wolf, ZRP $2007,44$.

4 Peemöller/Hofmann, Bilanzskandale, 2005.

5 Bannenberg, Korruption in Deutschland und ihre strafrechtliche Kontrolle, 2002, S. 375 m. w. N.

$6 \mathrm{Zu}$ den Problemen der Definition des Begriffs der Wirtschaftskriminalität vgl. Peemöller/Hofmann (Fn. 4), S. I7 ff; zum Umfang der in Frage kommenden Straftaten und auch Ordnungswidrigkeiten vgl. etwa Eidam, Unternehmen und Strafe 2 , $200 \mathrm{I}$.

7 Herbert/Oberrath, NZA 2005, 193 ff.; Müller, NZA 2002, $424 \mathrm{ff}$.

8 Vgl. Peemöller/Hofmann (Fn. 4), S. I 8 zur Bedeutung anonymer Strafanzeigen; Bannenberg/Schaupensteiner, Korruption in Deutschland, S. 40.

9 Vgl. dazu umfassend Eidam, S. 265 ff. (Fn. 6); vgl. auch Hauschka, NJW 2004, 257 ff; Bannenberg (Fn. 5), S. 2 I ff.

Io Zum Begriff des Rechtsgutes der Allgemeinheit vgl. m. w. N. Gänßle, Das behördliche Zulassen strafbaren Verhaltens, eine rechtfertigende Einwilligung?, 2003, S. $28 \mathrm{ff}$.

I I Rechtsgut ist die Planungs- und Dispositionsfreiheit der öffentlichen Hand in wirtschaftlicher Hinsicht, vgl. dazu Stöckel, ZRP I977, I 34 ff.; Lenckner, in: Schöncke/Schröder StGB, $\mathbb{2} 264$ RN 4; Tröndle/ Fischer, StGB, $\mathbb{} 264 \mathrm{RN}$ 3a jeweils m. w. N. 
298, ${ }^{12} 299^{13}$ oder 33 I ff. ${ }^{14}$ StGB verwiesen. Ohne Hinweise von Insidern, wie es Mitarbeiter eines Unternehmens sind, kommen sie schon deshalb kaum ans Licht der Strafverfolgungsbehörden, weil es an einem individualisierten Opfer fehlt, das nach erfahrener Rechtsgutverletzung selbst Strafanzeige erstatten könnte. Eine Verpflichtung von Unternehmen, entsprechende Sachverhalte nach interner Aufdeckung zur Anzeige zu bringen, besteht nicht. ${ }^{\text {Is }}$ Der Strafanzeige als »Bindeglied zwischen privater und hoheitlicher Deliktswahrnehmung « kommt daher eine besondere Bedeutung $\mathrm{zu}^{\mathrm{I}}{ }^{\mathrm{6}}$

Aus arbeitsrechtlicher Perspektive trifft der Whistleblower jedoch auf erhebliche Schwierigkeiten. Oft genug wird er infolge seiner Offenbarungen seinen Arbeitsplatz verlieren und um die wirtschaftliche Existenz fürchten müssen. ${ }^{17}$ Die arbeitsgerichtliche Rechtsprechung hält es grundsätzlich für zumutbar, dass sich der Whistleblower mit seinen Feststellungen zunächst an den Arbeitgeber wendet und so auf eine unternehmensinterne Aufklärung hinwirkt, bevor er Anzeige erstattet. Die aus kriminalpolitischer Sicht erwünschte Anzeige kann als rechtsmissbräuchliches und unverhältnismäßiges Vorgehen gegen den Arbeitgeber angesehen werden. Am Ende steht die zulässige verhaltensbedingte Kündigung des Mitarbeiters. ${ }^{\mathrm{I}}{ }^{8}$

Der folgende Beitrag beleuchtet das Spannungsverhältnis aus dem allgemeinen Interesse an Korruptionsbekämpfung, mithin dem Spannungsverhältnis aus kriminalpolitischen Wunschvorstellungen zur Bekämpfung von Wirtschaftskriminalität im Allgemeinen, ${ }^{19}$ und zivil- bzw. arbeitsrechtlichen Pflichten, in dem der Whistleblower steht. Die thematische Annäherung erfolgt mit dem Blick auf die strafrechtlichen Aspekte des Whistleblowing (2.). Die Argumente für (3.) und gegen (4.) eine verhaltensbedingte Kündigung im Fall der Anzeige des Arbeitgebers, ohne vorher eine unternehmensinterne Aufklärung angestrebt zu haben, werden ausgebreitet. Die differenzierte Betrachtungsweise, auf die das Urteil des Bundesarbeitsgerichts vom 3. 7. 2003-2 AZR 235/02 - verweist, wird erörtert (5.). Eine Reihe für die Praxis daraus folgender Fragen (6.) unterstreichen schließlich die Bedeutung unternehmensinterner Maßnahmen, die es einem möglichen Whistleblower erleichtern sollen, sich an Stelle einer Anzeigenerstattung unternehmensinternen Stellen zuzuwenden. Dabei wird der Beitrag zeigen, dass die Berücksichtigung von Möglichkeiten unternehmensinterner Offenbarung in der Rechtsprechung zu einem höheren Maß an Rechtssicherheit sowohl für den Arbeitgeber als auch für den Whistleblower führt (7.).

I 2 Schutzgut ist der freie Wettbewerb, vgl. dazu nur Tröndle/Fischer, StGB vor $\ 298$ RN 5; Kleinmann/ Berg, BB I 998, 277 ff.; dagegen eher den Schutz des Vermögens des Veranstalters im Vordergrund sehend Schaupensteiner, ZRP I993, $250 \mathrm{ff}$.

I 3 Zum geschützten Rechtsgut des freien Wettbewerbs vgl. Tröndle/Fischer, StGB, \299 RN 2 m. w. N.; SK-Rudolphi, StGB, $\ 299$ Rn. I.

I 4 Zum Schutz der Funktionsfähigkeit der Verwaltung bzw. der Lauterkeit des Handelns der Verwaltung und des entsprechenden Vertrauens darauf vgl. BGHSt I0, 237, 24I; I4, I 24, I 30; I 5, 88, 96; 30, 46, 48;

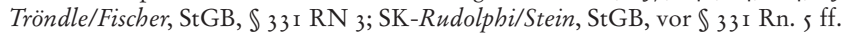

is Bannenberg (Fn. 5), S. 389 .

I6 Koch, NJW 2005, 943 ff.; vgl. Peemüller/Hofmann (Fn. 4), S. I8, wonach die nur zufällig ans Licht der Ermittlungsbehörden geratenen Fälle auf $44 \%$ beziffert werden; zur Problematik der Aufdeckung von Wirtschaftskriminalität vgl. auch Pelfini, Information Systems Audit and Controll Association (ISACA) NewsLetter April 2006, S. 5 ff.

I7 $\mathrm{Zu}$ den arbeitsrechtlichen Risiken von Whistleblowing vgl. nur Bannenberg (Fn. 5), S. 376.

I 8 Staudinger/Corts, \$626 RN 92; MK-Schwerdtner, \$626 RN 84; LAG Frankfurt/M., LAGE 28; LAG Ba-Wü NZA 1987, 756; LAG Berlin DB 1961, 576; LAG Düsseldorf BB 1959, 532; BAG NJW 2004, I $547 \mathrm{ff}$; Soergel ${ }^{12}-$ Kraft, $\ 626, \mathrm{RN} 53$.

I9 Zum Begriff der Wirtschaftskriminalität im Allgemeinen vgl. Fn. 6. 
Die strafrechtliche Betrachtung des Whistleblowing zeigt, dass derjenige, der eine schließlich begründete Anzeige einer Straftat oder Ordnungswidrigkeit vornimmt, ebenso vor strafrechtlicher Sanktion geschützt ist wie derjenige, dessen Anzeige einen Verdacht zugrunde legt, der sich schließlich nicht bestätigt. ${ }^{20}$ Auch ein Verfahrensausgang mit einer Verfahrenseinstellung nach $\mathbb{S} \mathbb{S}$ is $3 \mathrm{ff}$. StPO berührt den Anzeigenden nicht.

Eine Strafbarkeit wegen Falschverdächtigung nach $\mathbb{1}$ I64 StGB kommt nicht in Betracht. Die sogenannte Verdachtsanzeige bleibt nach $\$$ I64 StGB straflos. Ob sich der in der Anzeige erhobene Vorwurf schließlich bestätigt, ist unbeachtlich. ${ }^{21}$ Erforderlich ist lediglich, dass der Whistleblower ohne eine bestimmte Kenntnis von der Unwahrheit des im Anzeigevorwurf bezeichneten Sachverhaltes gehandelt hat. ${ }^{22}$ Auch eine Strafbarkeit wegen übler Nachrede nach $\$ I 86 StGB entfällt. Das Erstatten einer sogenannten Aufklärungsanzeige bei Vorhandensein entsprechend beachtlicher Anhaltspunkte erlaubt es dem Anzeigenerstatter, sich auf die Wahrnehmung berechtigter Interessen nach $\mathbb{\$}$ I93 StGB zu berufen. ${ }^{23}$ Wer seine Anzeige auf der Basis beachtlicher Anhaltspunkte stellt, vermag zudem durch die Information der Ermittlungsbehörden auch nicht den subjektiven Tatbestand des $\ 17$ UWG - Verrat von Geschäfts- und Betriebsgeheimnissen - zu erfüllen. Ihm wird es regelmäßig an einer der Formen besonderer Absicht fehlen, die der subjektive Tatbestand des $\mathbb{1 7}$ UWG fordert. ${ }^{24}$ Zusätzliche Motive für eine Anzeigenerstattung wie etwa Rachsucht, das Ziel, den anderen gleichsam »fertig zu machen « und anderes mehr spielen für die strafrechtliche Betrachtung des Whistleblowing in aller Regel keine Rolle. Strafrechtlich unbeachtlich ist es auch, ob der Anzeigenerstattung der Versuch einer unternehmensinternen Meldung und damit der Herbeiführung unternehmensinterner Aufklärung vorausgegangen ist. ${ }^{25}$ Dem arbeits- bzw. zivilrechtlichen Blick entspricht dieses Ergebnis - wie zu zeigen sein wird - jedoch nicht.

\section{Die Anzeige: ein Grund zur verhaltensbedingten Kündigung}

Im Arbeitsrecht kann die Erstattung einer Anzeige gegen den Arbeitgeber oder einen seiner Mitarbeiter einen Grund zur verhaltensbedingten Kündigung bie-

20 Vgl. Koch, NJW 2005, 944 .

2 I Die Verdachtsanzeige zur Aufklärung eines zweifelhaften Sachverhaltes (z.B. LK-Ruß, StGB, $\int$ I64 Rn. I3; Schönke/Schröder/Lenckner, StGB, $\mathbb{\int}{ }_{1}{ }_{4} \mathrm{RN}$ I 8$)$ ist von der Anzeige ins Blaue hinein zu unterscheiden (Tröndle/Fischer, StGB, $\mathbb{1}{ }_{6} 6_{4} \mathrm{RN}$ I 2.

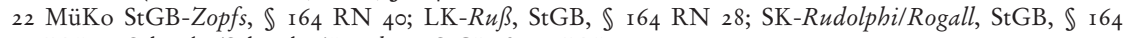
RN 40; Schönke/Schröder/Lenckner, StGB, $\mathbb{S}$ I 64 RN 30.

23 Bockelmann, NJW 1959, I849; Müller-Dietz, Tröndle-FS, S. 58 I; Tröndle/Fischer, StGB, \ 193 RN I 5.

24 Die Anzeigenerstattung geschieht nicht zu Zwecken des Wettbewerbs. Strafverfolgungsbehörden oder sonstige Ermittlungsbehörden sind keine Marktteilnehmer; vgl. dazu Kotthoff/Gabel, in: Ekey/Klippel/ Kotthoff/Meckel/Plaß, $\mathbb{I}$ I7 RN I4; Köbler, in: Baumbach/Hefermehl, $\mathbb{1}$ I7 RN 25 ; ein Handeln aus Eigennutz wird solange zu verneinen sein, wie es dem Anzeigeerstattenden darum geht, tatsächlich ein Strafverfahren einzuleiten und er es nicht bewußt unterlässt, sich selbst um die Sachverhaltsaufklärung zu bemühen; zum immateriellen Vorteil vgl. BGHSt I I, 97; sowie auch RGSt 9, I66 ff; I6, 93 ff; 29, 426 ff; 5 I, I 84 ff; Harte-Bavendamm, in: Gloy/Loschelder, HdB des Wettbewerbsrechts 3 , $\$ I7 RN 26. Es reicht allein zudem nicht aus, dem Unternehmer mit der Anzeigenerstattung Schaden zufügen zu wollen; vgl. dazu Köhler in Baumbach/Hefermehl, $\mathbb{S}$ I7 RN 26; Harte-Bavendamm in Gloy/Loschelder, ebd., $\mathbb{S} 7$ RN 27. Es liegt auch kein Handeln zugunsten eines Dritten vor; vgl. BT-Drs. I0/5058, S. 40; a. A. dazu

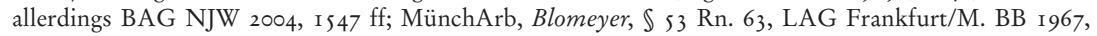
I 68 .

25 Koch, NJW 2005, 943 ff; vgl. Bockelmann, NJW I959, I 849 ff.; BGHSt I I, 97. 
ten. Voraussetzung dafür ist, dass die Anzeige als rechtsmissbräuchliches oder wenigstens unangemessenes Vorgehen gegenüber dem Arbeitgeber zu deuten ist. ${ }^{26}$ Damit ist die zivilrechtliche Sanktionierung einer erstatteten Anzeige praktisch mindestens ebenso bedeutsam wie eine gedachte strafrechtliche Sanktion. Insbesondere bei Anzeigen, die sich gegen das Verhalten von Mitarbeitern im Unternehmen wenden, wird ein unangemessenes Vorgehen bereits bejaht, wenn der Anzeigende es nur unterlassen hat, vor Anzeigenerstattung auf eine unternehmensinterne Aufklärung hinzuwirken. ${ }^{27}$ Berücksichtigt man, dass heute eine große Zahl von Arbeitgebern juristische Personen des Privatrechts sind, hat die grundsätzliche Forderung nach dem Vorrang einer unternehmensinternen Aufklärung eine wesentliche Bedeutung für das Whistleblowing. Gibt es einen personifizierbaren Arbeitgeber nicht mehr, richtet sich eine mögliche Anzeige stets gegen irgendwie zu bestimmende »Mitarbeiter « des Arbeitgebers, und sei es gegen Geschäftsführer und Vorstandsmitglieder selbst.

Grundlage für die Forderung nach vorrangiger unternehmensinterner Aufklärung ist das Rücksichtnahmegebot, wie es heute in $\ 24 \mathrm{I}$ Abs. 2 BGB gefasst ist. ${ }^{28}$ Der Mitarbeiter hat danach die Pflicht, Schäden von seinem Arbeitgeber abzuwenden bzw. sich so zu verhalten, dass die Interessen des Arbeitgebers nicht verletzt werden. ${ }^{29}$ Zudem wird auf Regelungen aus dem Arbeitsschutz verwiesen. Dort hat sich der Mitarbeiter bei Verstößen gegen Arbeitsschutzvorschriften zunächst an den Arbeitgeber zu wenden, bevor er die zuständigen Behörden informieren darf. $3^{\circ}$ Regelungen wie etwa $\ 2$ I Abs. 6 GefahrstoffVO, $\ 30$ Abs. I StrlSchVO oder $\int$ I 6 ArbSchG sehen vor, dass der Mitarbeiter sich bei Störungen oder Verstößen zuerst intern an seinen Arbeitgeber zur Abhilfe zu wenden hat. Was im Bereich des Arbeitsschutzes gilt, soll ein auf jeden Fall möglicher Anzeigenerstattung übertragbarer Grundsatz sein. ${ }^{31}$

Der Arbeitgeber verdient es insbesondere, davor geschützt zu werden, dass ein Mitarbeiter durch leichtfertig falsche Angaben bei der Anzeigenerstattung ihm wie auch seinem Unternehmen Schaden zufügt. Jede Anzeige ist, gleich ob schließlich begründet oder nicht, geeignet, wenigstens einen nicht unerheblichen Imageschaden für das Unternehmen und damit auch für die Position des Unternehmens am Markt und im Wettbewerb herbeizuführen. ${ }^{22}$ Die Priorität einer unternehmensinternen Vorgehensweise würde es dem Arbeitgeber erlauben, in wirtschaftlich effektiver Form zu handeln, die Vertiefung bereits eingetretener Schäden zu verhindern sowie drohende Schäden vom Unternehmen abzuwenden. 33

Zusätzlich soll es auch auf die Motivation des Anzeigeerstattenden ankommen. Wer Strafanzeige stellt und sich beispielsweise damit an seinem Arbeitgeber für dessen vergangenes Verhalten rächen möchte, bleibt zwar vor strafrechtlicher Sanktion bewahrt, aus arbeitsrechtlicher Sicht liegt aber ein rechtsmissbräuchliches Vorgehen vor. 34 Der Mitarbeiter muss sich nunmehr entgegenhalten lassen,

26 So heute die Formel in BAG NJW 2004, I 547 ff.; vgl. auch schon BAG BB 1959, 920; LAG Düsseldorf BB I960, 523; LAG Düsseldorf BB I96I, 532; auch LAG Ba-Wü Urt. v. 20.10.1976, EzA \ I KSchG Verhaltensbedingte Kündigung Nr. 99; LAG Hamm DB 2004, 442 f; LAG Berlin BB I96 I, 449 f.

27 BAG BB I959, 920; MünchArbR, Blomeyer, $\ 52$ RN I; neuerlich noch einmal BAG NJW 2004, I $547 \mathrm{ff}$.

28 Vgl. dazu Herbert/Oberrath, NZA 2005, I93, I95; BAG NJW 2004, I 547 ff.

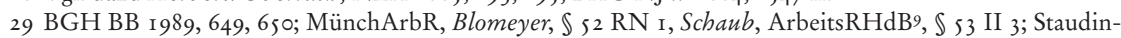
ger/Preis, Buch 2, 2002, $\$ 626 \mathrm{RN}$ I 3 I ff.

30 AsiR, $\mathbb{1} 16 \mathrm{RN}_{4}$ f; Gach/Rützel, BB I997, I 959 ff; MK-Schwerdtner $\$ 626 \mathrm{RN} 85$.

3 I Dazu Gach/Rützel, BB i997, I959, I96 I f.

32 Bannenberg (Fn. 5), S. 385 .

33 MünchArbR, Blomeyer, $\int 52$ RN I; Schaub, ArbeitsRHdB9, $\$ 53$ II 3; das aufgreifend BAG NJW 2004, I 547.

34 BAG NJW 2004, I 547 ff; dazu auch Gach/Rützel, BB I997, I959 ff. 
dass ihm ein schutzwürdiges Interesse an der Erstattung einer Anzeige entweder gänzlich fehlt oder aber zumindest nur noch eine so untergeordnete Rolle spielt, dass er sich darauf aus zivil- bzw. arbeitsrechtlicher Sicht nicht mehr berufen kann. 35 Dem Arbeitgeber wird die Fortsetzung des Arbeitsverhältnisses mit einem Mitarbeiter, der ihn durch das Erstatten von Strafanzeigen zu schädigen sucht, kaum mehr zumutbar sein - und zwar ungeachtet der Begründetheit der Strafanzeige. ${ }^{36}$

Während also aus strafrechtlicher Sicht das Erstatten einer Anzeige gegen den Arbeitgeber oder dessen Mitarbeiter für den Anzeigeerstattenden auch dann ohne Sanktion bleibt, wenn eine vorhergehende unternehmensinterne Aufklärung nicht angestrebt worden ist oder die Anzeigenerstattung von persönlichen Rachegefühlen des Anzeigeerstatters begleitet war, hat der Whistleblower aus zivil- bzw. arbeitsrechtlicher Perspektive nunmehr mit dem Verlust seines Arbeitsplatzes infolge einer verhaltensbedingten Kündigung zu rechnen. Diese kann im Übrigen sogar fristlos erfolgen.37 Auf die Wahrnehmung berechtigter Interessen mit der Erstattung der Anzeige kommt es dann nicht mehr an. Ebensowenig kommt es auf die Begründetheit der Anzeige an. Die zivil- bzw. arbeitsrechtliche Betrachtung rückt allein die Frage nach der Zumutbarkeit der Fortsetzung des Arbeitsverhältnisses aus der Sicht des Arbeitgebers nach der Anzeigenerstattung in den Fokus. ${ }^{3}$ Die entsprechende Abwägung zugunsten des Arbeitgebers findet auf der Basis des Gebotes der gegenseitigen Rücksichtnahme zweier Vertragsparteien auf die jeweils auf der anderen Seite bestehende Interessenlage statt.

\section{Die Anzeige: kein Grund zur verbaltensbedingten Kündigung}

Die Forderung nach der Priorität unternehmensinterner Aufklärung vor Anzeigenerstattung ist nicht ungeteilt. So darf die Möglichkeit der Anzeigenerstattung auch als Bestandteil der nach Art. 2 Abs. I GG grundrechtlich geschützten Handlungsfreiheit angesehen werden.39 Weiter soll das individuelle Recht zur Anzeigenerstattung gegen den Arbeitgeber bzw. seine Mitarbeiter aus dem Petitionsrecht ableitbar sein. $4^{\circ}$ Eine nicht anonym erstattete Anzeige kann zudem unter den Schutz der Meinungsfreiheit nach Art. s Abs. I GG fallen. ${ }^{4}{ }^{\mathrm{I}}$ Das Recht des Mitarbeiters, gegen seinen Arbeitgeber bzw. dessen Mitarbeiter Anzeige zu erstatten, soll mithin unmittelbar grundrechtlich verankert sein.

$\mathrm{Zu}$ den staatlichen Aufgaben gehört es zudem, das friedliche Zusammenleben der Bürger untereinander zu sichern. Rechtssicherheit ist dazu neben der Freiheit zur individuellen Lebensgestaltung eine unbedingte Voraussetzung. Das staatliche Gewaltmonopol verbietet es dem Einzelnen, selbst dem Recht zur Durchsetzung zu verhelfen. Ausnahmen, die im Wesentlichen gesetzlich geregelt sind, bestehen freilich im Bereich von Notwehr, Nothilfe, Selbsthilfe und

35 BAG, Urt. v. 05.02.1959, EzA $\ 70$ HGB Nr. I; BAG, Urt. v. 4.7.199I, RzK I 6 Nr. 74; BAG DB I970, I739; LAG Hamm BB i950, 703.

36 Vgl. nur BAG NJW 2004, I 547 ff; BAG, Urt. v. 05.02.1959, EzA \70 HGB Nr. I; BAG, Urt. v. 4.7.1991, RzK I 6 Nr. 74.

37 BAG NJW 2004, I $547 \mathrm{ff}$.

38 Soergel-Kraft, $\$ 626 \mathrm{RN} 53$.

39 BVerfG NJW 200I, 3474 ff; schon zuvor BVerfGE 74, 257 ff.

40 Staudinger/Preis, Buch 2, 2002, $\mathbb{6} 626$ RN I3 I; BGB-RGRK Corts, $\mathbb{S} 626$ RN 92, LAG Frankfurt/M. BB I 987, I 320.

4I Müller, NZA 2002, 424, 429 f; ErfK-Dietrich, Art 5, RN 37. 
der vorläufigen Festnahme..$^{2}$ Von diesen Ausnahmen abgesehen, ist der Einzelne gehalten, sich bei erkannten vermeintlichen oder tatsächlichen Rechtsverletzungen an die für die Beachtung des maßgeblichen Rechts zuständigen staatlichen Stellen zu wenden. Entsprechendes Verhalten liegt im allgemeinen öffentlichen Interesse. 43 Weil also das Verbot der privaten Rechtsdurchsetzung den Staat verpflichtet, für die Rechtssicherheit Sorge zu tragen, muss dem einzelnen Bürger die Möglichkeiten gegeben sein, Anzeige gegen den Arbeitgeber bzw. dessen Mitarbeiter frei von staatlichen Sanktionen stellen zu können, das heißt nicht nur frei von strafrechtlichen Sanktionen. Es soll vielmehr darüber hinaus im Rahmen der Rechtsanwendung bei der Auslegung der Reichweite des Rücksichtnahmegebotes nach $\$ 24$ I Abs. 2 BGB mit Rücksicht auf den besonderen grundrechtlichen Schutz des Anzeigeerstattens auch eine arbeitsrechtliche Sanktion des Anzeigeerstattenden ausbleiben. Zudem darf kein Vertrauensschutz des Arbeitgebers hinsichtlich objektiv rechtlich zu missbilligendem und mithin nicht schutzwürdigem Verhalten entstehen. ${ }^{44}$ Strafrechtlich relevantes Verhalten würde sonst unter arbeitsrechtlichen Schutz gestellt werden.45

Der Verweis auf die Treuepflicht - heute das Rücksichtnahmegebot gemäß $\$ 24 \mathrm{I}$ Abs. 2 BGB - als Grund für eine Priorität unternehmensinterner Aufklärung gegenüber externer Anzeigenerstattung führt zudem zu einer im Schuldrecht bei der Betrachtung von Parteiinteressen in Leistungsbeziehungen sonst nicht anzutreffenden automatischen Bevorzugung einer von zwei Parteien. ${ }^{46}$ In der Festschreibung des Rücksichtnahmegebotes in \$24I Abs. 2 BGB wurde lediglich ein allgemeines Grundprinzip zum Ausdruck gebracht. Partner vertraglicher Beziehungen haben danach beim Vollzug einer zwischen ihnen getroffenen Vereinbarung für ein gedeihliches Miteinander einzustehen. Schutzgut ist das jeweilige Integritätsinteresse einer Partei.47 Mag das auch Aufklärungspflichten und Pflichten zur Schadensabwendung des Mitarbeiters gegenüber seinem Arbeitgeber begründen, verbietet das in $\$ 24 \mathrm{I}$ Abs. $2 \mathrm{BGB}$ gefasste Rücksichtnahmegebot nicht schon aus sich selbst heraus die Anzeigenerstattung ohne den Versuch vorhergehender unternehmensinterner Aufklärung. Der Gehalt des Rücksichtnahmegebotes vermag nur soweit zu reichen, wie er mit Rücksicht auf die Wirkung unterschiedlicher Grundrechtsinteressen, die im Einzelfall einer Anwendung von $\ 24 \mathrm{I}$ Abs. 2 BGB zum Ausgleich zu bringen sind, bestimmt werden kann..$^{8}$ Ein Vorrang unternehmensinterner Sachverhaltsaufklärung besteht danach in Ansehung strafbarer oder ordnungswidriger Verhaltensweisen, die durch Nichtanzeige gedeckt würden, nicht mehr ohne weiteres. Er müsste vielmehr aus einer Interessenabwägung zwischen dem Interesse des Anzeigeerstattenden an der Anzeigenerstattung und dem Interesse des Arbeitgebers am Schutz seines eingerichteten und ausgeübten Gewerbetriebes ermittelt werden.49

Die verhaltensbedingte Kündigung ließe sich dann etwa beim Vorliegen einer verwerflichen Motivation des Anzeigeerstatters immer noch rechtfertigen. Das-

42 Darauf verweisend BAG NJW 2004, I 547 , I 549.

43 LAG Ba-Wü NZA 1987, 756; dazu Anm. v. Colneric, AiB 1987, 260; vgl. auch schon Dahns, BB I949, $39 \mathrm{ff}$.

44 Staudinger/Preis, Buch 2, 2002, $\$ 626$ RN I3 I.

45 Darauf im Ergebnis verweisend MK4-Henssle, $\ 626$ RN I67; dazu auch Preis, DB I988, I444, I448; zum

"Selbstwiderspruch der Rechtsordnung v vgl. Rützel, GRUR I995, $557 \mathrm{ff}$.

$46 \operatorname{Kempf,DB}$ i $979,790 \mathrm{ff}$ m. w. N.

47 Palandt-Heinrichs ${ }^{65}, \ 24$ I RN 6 ff.

48 Zur Bestimmung des Inhaltes der Rücksichtnahmepflicht unter Bezugnahme auf die Grundrechte vgl. Herbert/Oberrath, NZA 2005, I93, I95 m. w. N.; auch BAG NJW 2004, I 547 ff m. w. N.

49 Zum grundrechtlichen Schutzgut des Arbeitgebers, vgl. BAG NJW 2004, I 547 , I 549. 
selbe würde auch angesichts völlig haltloser Vorwürfe gelten. ${ }^{\circ}$ Auch wenn der unternehmensinternen Aufklärung kein Vorrang eingeräumt würde, stünde die Anzeige also noch unter einem Missbrauchsvorbehalt. Die Wahrnehmung berechtigter Interessen des Anzeigenden wäre darauf beschränkt, durch die Anzeigeerstattung nicht die Verächtlichmachung des Arbeitgebers - und sei es nur als nicht unerwünschte Nebenfolge - anzustreben. ${ }^{\text {I }}$ Wiederum würde aber der zivil- bzw. arbeitsrechtliche Schutz des Whistleblowers nicht so weit reichen wie der strafrechtliche Schutz.

\section{Eine differenzierte Betrachtungsweise}

Die Rechtsprechung des Bundesarbeitsgerichts hat in jüngerer Zeit unter Rückgriff auf die Rechtsprechung des Bundesverfassungsgerichts ${ }^{2}$ eine Anzeige gegen den Arbeitgeber bzw. dessen Mitarbeiter für rechtlich zulässig erachtet, wenn dem Anzeigenden die Herbeiführung einer innerbetrieblichen Aufklärung unzumutbar ist, weils3

- der Anzeigende bei Nichtanzeige sich gemäß $\int{ }_{3} 8$ StGB selbst der Strafverfolgung aussetzen würde,

- die angezeigte Straftat von besonderer Schwere ist,

- vom Arbeitgeber selbst begangen worden ist,

- Abhilfe im Fall unternehmensinterner Meldung berechtigter Weise nicht zu erwarten ist oder

- der Versuch, unternehmensinterne Abhilfe herbeizuführen, erfolglos geblieben ist. 54

Eine vorrangige Pflicht zur unternehmensinternen Meldung soll auch dann nicht bestehen, wenn der maßgebliche Verstoß dem Arbeitgeber bekanntss oder grob fahrlässig unbekannt war ${ }^{5}$ oder wenn der Umstand mit großer Wahrscheinlichkeit dem Arbeitgeber bekannt sein musste.57 Denn der Arbeitgeber darf nur so lange darauf vertrauen, dass ein zu missbilligendes Verhalten nicht nach außen bekannt wird, solange er selbst keine Kenntnis davon hat und deshalb nicht selbst für Abhilfe sorgen kann. ${ }^{8}$

Hinzu kommen in der Rechtsprechung Fälle, in denen durch die Anzeige Gefahren für Leib und Leben Dritter abgewendet worden sind - etwa im Fall der Anzeige eines Verstoßes gegen lebensmittelrechtliche Vorschriften.59 Allerdings wird von der insoweit vorliegenden arbeitsgerichtlichen Rechtsprechung lediglich der Vorrang unternehmensinterner Aufklärung zurückgenommen. Ist die Erstattung der Anzeige ferner mit verwerflichen Motiven wie Rachsucht oder dergleichen mehr verknüpft, soll ungeachtet der vorliegenden Ausnahmetatbestände eine verhaltensbedingte Kündigung möglich bleiben. ${ }^{60}$

50 Soergel-Kraft, $\$ 626$ RN 53; LAG Frankfurt/M. BB I987, I $320 \mathrm{f}$.

5 I Staudinger/Preis, Buch 2, 2002, $\$ 626 \mathrm{RN}$ I3 I.

52 BVerfG NJW 200I, $3474 \mathrm{ff}$.

53 BAG NJW 2004, I 547 , I 550.

54 Vgl. a. Preis/Reinfeld, AuR I989, 36 I, 372; MünchArbR, Blomeyer $\int 5$ I RN 7I.

55 LAG Ba-Wü NZA I987, 656; MünchArbR, Blomeyer, $\mathbb{S} 52$ RN 52; vgl. a. Gach/Rützel, BB I997, I959, I96I.

56 Preis/Reinfeld, AuR i989, 361, 370.

57 MK3-Söllner, $\mathbb{6} 6$ I $\mathrm{RN} 398$.

58 Erman $^{10 / B e l l i n g, ~} \$ 626 \mathrm{RN} 55$.

59 LAG Ba-Wü NZA $1987,756 \mathrm{f}$.

60 BAG NJW 2004, I 547 ff; LAG Frankfurt/M. BB 1987, I320; Staudinger/Preis, Buch 2, 2002, $\mathbb{S} 626$ RN i3 If. 
Die unterschiedliche Behandlung des Whistleblowing im Strafrecht und dem Zivil- bzw. Arbeitsrecht verdient im Grundsatz ihre Zustimmung. Die strafrechtliche Sanktion ist ein staatlicher Eingriff, der stets ultima ratio bleiben muss. Was strafrechtlich deshalb nicht beachtlich sein soll, muss von der Rechtsordnung an anderer Stelle noch nicht gebilligt werden. Bleibt der Whistleblower also straflos infolge einer Anzeigenerstattung, muss die Anzeigenerstattung nicht automatisch zivil- bzw. arbeitsrechtlich folgenlos bleiben.

In der Praxis wird es aber oftmals eine nur schwer zu beantwortende Frage sein, wann dem Whistleblower die innerbetriebliche Meldung unzumutbar war. Für ihn ist es im Voraus kaum zu beurteilen, wann er auf einen ihm möglichen unternehmensinternen Hinweis berechtigter Weise nicht mit Abhilfe zu rechnen braucht. Erst recht vermag der Whistleblower es nicht zu beurteilen, ob - wie von Preis/Reinfeld vertreten ${ }^{6 \mathrm{I}}$ - beim Arbeitgeber möglicherweise fahrlässige Unkenntnis vorliegt, bzw. der maßgebliche Umstand überhaupt bekannt ist. Muss der Whistleblower zudem die innerbetriebliche Meldung bzw. Aufklärung selbst gar mit gewisser Intensität vorantreiben? Müssen also auch dann noch weitere Versuche zur Herbeiführung einer unternehmensinternen Klärung unternommen werden, wenn der Whistleblower auf seinen Hinweis nur die Antwort erhält, es sei schon alles von oben gedeckt? Wer im Unternehmen kann in einem solchen Fall noch der richtige Adressat eines Hinweises sein? Diese Frage stellt sich insbesondere dort, wo der Arbeitgeber lediglich durch Organe juristischer Personen des Privatrechts bzw. deren Mitglieder vertreten wird.

Das Bundesarbeitsgericht hat es in einem Fall nicht ausreichen lassen, dass der Anzeigenerstatter sich zunächst an seinen Vorgesetzten gewandt hatte, dann zwei Jahre später diesem den noch immer andauernden Sachverhalt erneut vorgehalten hat, um dann schließlich einige Monate später selbst Strafanzeige zu erstatten. ${ }^{62}$ Bei der ersten Meldung an den Vorgesetzten drohte dieser dem Hinweisgeber und bedeutete, dass alles von oben gebilligt sei. Deshalb unterblieb eine weitere Meldung an einen höheren Vorgesetzten. Der Hinweisgeber glaubte jedoch, durch seine Tätigkeit fortlaufend einen Subventionsbetrug seines Arbeitgebers zu unterstützen. Das war für ihn Anlass zu einem zweiten Vorgehen und schließlich für die Anzeigenerstattung bei der Staatsanwaltschaft. Ist es dem Mitarbeiter in gewissen Grenzen zumutbar, das Risiko, sich selbst strafbar zu verhalten, einzugehen, nur weil er auf das Ergebnis einer unternehmensinternen Meldung eines strafrechtlich relevanten Sachverhaltes zu warten hat? Unklar ist auch, wie schnell der Whistleblower nach seinem Meldeversuch Strafanzeige erstatten muss. Im hier beschriebenen Fall lagen zwischen dem letzten Klärungsversuch und der Anzeigenerstattung zumindest ein paar Monate. Zudem bestand im Zeitpunkt der Anzeigenerstattung noch ein Konflikt zwischen dem Hinweisgeber und seinem Vorgesetzten, gegen den sich zugleich die Anzeige richtete. ${ }^{63}$ Doch muss daraus auch schon eine verwerfliche Motivation zur Anzeigenerstattung folgen?

Bereits die gegenüber den Ermittlungsbehörden anonyme Anzeigenerstattung soll Rückschlüsse auf ein unverhältnismäßiges und unangemessenes Vorgehen des Anzeigenden zulassen dürfen. ${ }^{64}$ Der Schutz der Anonymität soll dem Whist- 
leblower einerseits die Möglichkeit geben, einen rechtswidrigen Zustand durch Ingangsetzen staatlicher Ermittlungen zu beenden, ohne sich selbst andererseits der unmittelbaren Gefahr des Arbeitsplatzverlustes und der Gefährdung seiner wirtschaftlichen Existenz auszusetzen. Daher wird aus kriminalpolitischer Sicht der anonymen Anzeige eine wesentliche Bedeutung bei der Bekämpfung von Wirtschaftskriminalität gegeben. ${ }^{65}$ Die arbeitsgerichtliche Rechtsprechung hält dem Whistleblower aber gerade sein anonymes Vorgehen entgegen und führt im Zweifel genau zum gegenteiligen Ergebnis: Arbeitsplatzverlust durch verhaltensbedingte Kündigung.

Insgesamt kann man mit Rücksicht auf die aufgeworfenen Fragen ein Konzept des arbeitsrechtlichen Schutzes von Whistleblowern selbst dann kaum noch erkennen, wenn die Anzeigenerstattung nicht von zusätzlichen verwerflichen Motiven wie Rachsucht oder dergleichen mehr getragen ist. Die Aufforderung, sich zunächst um eine unternehmensinterne Sachverhaltsklärung zu bemühen, ist für den Whistleblower regelmäßig mit dem Risiko nicht unerheblicher Repressalien durch den Arbeitgeber verbunden. ${ }^{66}$ Dort, wo der Arbeitgeber als juristische Person durch entsprechende Organe und deren Mitglieder vertreten ist, verweist der Vorrang der unternehmensinternen Meldung den Mitarbeiter nur an seine fachlichen bzw. disziplinarischen Vorgesetzten.67 Gerade diese könnten aber selbst in den maßgeblichen Sachverhalt verstrickt sein oder auch nur das Fehlverhalten übergeordneter Manager decken wollen. ${ }^{68}$ Allein durch die Betrachtung im Nachhinein bei der gerichtlichen Auseinandersetzung um die Rechtmäßigkeit der ausgesprochenen Kündigung vermag der Whistleblower Gewissheit über die Rechtmäßigkeit seines Vorgehens zu erhalten. Das Arbeitsverhältnis wird gleichwohl oft genug so zerrüttet sein, dass nur noch seine Beendigung, wenn auch mit einer Abfindung, bleibt. Der Whistleblower ist damit hinsichtlich seines Schutzes auf die richterliche Rechtsfortbildung bei der Anwendung von \24I Abs. 2 BGB sowie des Kündigungsschutzgesetzes angewiesen. ${ }^{69}$

Im Zweifel führt eine derartige Situation nicht dazu, dass strafrechtlich relevante oder dem Ordnungswidrigkeitenrecht zuzurechnende Sachverhalte zur Verfolgung durch die zuständigen Behörden gelangen. Die Folge ist vielmehr, dass gerade Straftaten und Ordnungswidrigkeiten, die zu einem hohen wirtschaftlichen Schaden führen, in zahlreichen Fällen unentdeckt bleiben. ${ }^{\circ}$ Das entspricht keineswegs dem sich in der öffentlich geführten Diskussion um Korruptionsbekämpfung und Prävention von Straftaten und Ordnungswidrigkeiten widerspiegelnden Interesse an ihrer Bekämpfung im Zuge unternehmerischen Handelns. ${ }^{71}$ Es entspricht aber auch nicht dem Interesse des Unternehmens. ${ }^{72}$

65 Vgl. Fn. 9; Koch, NJW 2005, $943 \mathrm{ff.}$

66 Vgl. dazu etwa Bannenberg (Fn. 5), S. 376.

67 Vgl. dazu nicht zuletzt auch BAG NJW 2004, I $547 \mathrm{ff}$.

68 Bannenberg, Wirtschaftskriminalität und Korruption, BKA Herbsttagung 2002, 2003, S. 43 ff.

69 Den gesetzlichen Schutz in Deutschland insoweit als unzureichend bezeichnend Röhrbein, ZIR 2004, 27 I.

70 Angaben zu tatsächlichen Schadenshöhen sind freilich nur schwer zu verifizieren; vgl. i. E. dazu Stierle, ZIR 2006, I09 ff.; Salvenmoser, ZIR 2007, 2 ff.; vgl. zu Schadenshöhen auch die Beispiele bei Peemöller/ Hofmann (Fn. 4), insbes. S. 80 ff.; bzgl. Submissionsabsprachen vgl. Greeve, Korruptionsdelikte in der Praxis, 2005, RN 507 mit Verweis auf BGH NJW 1992, 92 I ff.; Preisabsprachen in der Bauwirtschaft sollen lt. BGH BauR I 996, 384 ff. zu bis zu I3\% überhöhten Preisen führen.

7I Vgl. dazu Hauschka, NJW 2004, 257 ff.; Lampert, BB 2002, 2237 ff.; von Rosen, Der Konzern 2004, 325; Bundesverband der Deutschen Industrie, Korruption verhindern - Empfehlungen des BDI, 2. Auflage.

72 Ausführlich dazu Stierle, ZIR 2006, I09, I I I. 
Neben dem hohen volkswirtschaftlichen Schaden, der mit den hier angesprochenen Straftaten oder Ordnungswidrigkeiten verbunden ist, können aber auch für die betroffenen Unternehmen hohe Geldbußen und Schadensersatzforderungen aus Straftaten oder Ordnungswidrigkeiten ihrer Mitarbeiter resultieren.73 Unternehmen haben daher selbst ein Interesse, Straftaten und ordnungswidriges Handeln ihrer Mitarbeiter zu unterbinden bzw. aufzudecken.74 Hinzu kommt, dass die ordnungsmäßige Geschäftstätigkeit heute als fester Bestandteil der Corporate Governance-Grundsätze wirtschaftlichen Handelns erwartet wird.75 Es entstehen zudem nicht geringe persönliche Haftungsrisiken der Mitglieder von Vertretungsorganen. ${ }^{76}$ Geeigneten unternehmenseigenen Präventionsmaßnahmen kommt mithin eine hohe Bedeutung zu. Für die effektive Wirksamkeit von Präventionsmaßnahmen wird der Schutz von Interessen möglicher Hinweisgeber bedeutsam sein.77 Präventionsmaßnahmen gegen Straftaten und Ordnungswidrigkeiten müssen sich daran messen lassen, ob sie eine Brücke schlagen zwischen dem Interesse an der Entdeckung einer Straftat einerseits und den aus dem Rücksichtnahmegebot nach $\mathbb{S} 24$ I Abs. 2 BGB folgenden Pflichten eines Mitarbeiters andererseits. Wer beim Aufdecken eines strafrechtlich relevanten Fehlverhaltens um seinen Arbeitsplatz fürchten muss, wird am Ende lieber schweigen als sein Wissen offenbaren.

Präventionsmaßnahmen sind in vielfältiger Form vorstellbar. Sie betreffen zum einen unternehmenseigene Prozesse und bilden sich in internen Kontrollsystemen ab. ${ }^{7}$ Derartige prozedurale Präventionsmaßnahmen gewinnen insbesondere vor dem Hintergrund der Organhaftung ihre Bedeutung. Die Mitglieder der Organe einer $\mathrm{GmbH}$ oder Aktiengesellschaft etwa haben im Rahmen ihrer Pflichten zu einer sicheren Unternehmensführung geeignete Maßnahmen zur Gewährleistung der kaufmännischen Sicherheit unternehmensinterner Prozesse zu ergreifen. 79 Neben solchen prozeduralen Präventionsmaßnabmen sind auch solche vorstellbar, die hier als institutionelle Präventionsmaßnabmen bezeichnet werden sollen.

Institutionelle Präventionsmaßnahmen führen dazu, unternehmensinterne Institutionen zu nutzen, zu schaffen oder auszubauen, die geeignet sind, Hinweise von möglichen Straftaten oder Ordnungswidrigkeiten aufzunehmen, zu prüfen, maßgebliches Verhalten zu beenden und gegebenenfalls Anzeigen gegen Mitarbeiter des Unternehmens zu erstatten. Wesentliche institutionelle Präventionsmaßnabmen sind etwa die Einrichtung einer Innenrevision, ${ }^{80}$ sogenannter Kor-

73 Lampert, BB 2002, $2237 \mathrm{ff}$.

74 Vgl. dazu von Rosen (Fn. 7I), S. $325 \mathrm{ff}$.

75 Lampert, BB 2002, 2237 ff; Bundesverband der Deutschen Industrie, Korruption verhindern (Fn. 7I).

76 Hierauf verweisend Hauschka, NJW 2004, $257 \mathrm{ff}$.

77 Vgl. Fn. 9.

78 Zur Definition interner Kontrollsysteme vgl. van den Brink/Romeike, Corporate Governance und Risikomanagement im Finanzdienstleistungsbereich, 2005, S. 3 ff; Standard Nr. 3 des Instituts für Interne Revision (IIR), S. I4, am 19.06.2007 unter www.iir-ev.de/deutsch/download/Revisionsstandard_ Nr._3.pdf;

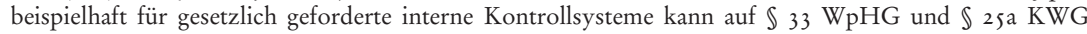

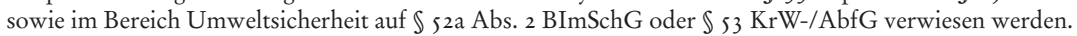

79 Hauschka, NJW 2004, 26I.

80 Bannenberg (Fn. 5), S. 25 I; Hauschka, NJW 2004, 257, 260; zu einem Wandel der Innenrevision über ihr klassisches Bild hinaus auch im Hinblick auf die Verfolgung von strafrechtlich relevanten und ordnungswidrigen Verhaltensweisen vgl. Peemöller, in: Fröschle/Peemöller, Wirtschaftsprüfung und Interne Revision, 2004, S. I I ff.; Janke, BUW 2003, I0I3; Lück/Henke, BfuP 2004, I ff.; Risak, ZIR 2004, 98 ff.; Knapp, Interne Revision und Corporate Governance, 2005, S. $37 \mathrm{ff}$. 
ruptions- bzw. Antikorruptionsbeauftragter, ${ }^{81}$ Compliancebeauftragter ${ }^{82}$ und

Ombudsleute $^{8}$ oder Korruptionscontroller. $^{84}$ Insbesondere Letztere können zugleich Mitarbeiter des Unternehmens oder externe, vom Arbeitgeber entsprechend Beauftragte sein. ${ }^{85}$ Das Bestehen solcher Stellen, an die sich der Whistleblower wenden kann, erlaubt es ihm, seine Wahrnehmungen außerhalb der disziplinarischen Hierarchien der eigenen funktionalen Eingebundenheit im Unternehmen zu kommunizieren. Präventionsmaßnahmen jeder Art ist gemeinsam, dass sie die Begehung möglicher Straftaten oder Ordnungswidrigkeiten erschweren, indem sie insbesondere das Entdeckungsrisiko erhöhen. ${ }^{86}$ Im Ergebnis gilt das für die Festlegung der Erforderlichkeit einer zusätzlichen Unterschrift ebenso wie etwa für die Einrichtung einer Innenrevision oder eines Antikorruptionsbeauftragten. Die Motivation eines Unternehmensprozedurale oder institutionelle Präventionsmaßnabmen $\mathrm{zu}$ entwickeln, wird daher auch stets dieselbe sein. Es ist der Wunsch der Unternehmensführungen, rechtstreues Verhalten der Mitarbeiter sicherzustellen und wirtschaftliche Schäden, aber auch Imageschäden vom Unternehmen abzuwenden. ${ }^{87}$

Ob Präventionsmaßnahmen ergriffen worden sind oder nicht, wird bei der Strafzumessung von Bedeutung sein, wenn es darum geht, die kriminelle Energie des Täters zu bewerten. Dabei gelangen dort insbesondere die hier als prozedural bezeichneten Präventionsmaßnabmen in den Fokus der Betrachtung. Ihr Vorhandensein bestimmt unmittelbar darüber, wie leicht einem Täter die Tatbegehung gemacht wird. ${ }^{88}$ Etwa wird das Erfordernis einer zweiten Unterschrift eine mögliche Untreuehandlung gegenüber einer Einzelzeichnungsberechtigung regelmäßig erschweren.

Das Vorhandensein institutioneller Präventionsmaßnabmen kann dagegen insbesondere für die arbeitsrechtliche Betrachtung des Whistleblowing zunehmende Bedeutung erlangen. Ein Unternehmen, das Anlaufstellen für Mitarbeiter zur Abgabe von Hinweisen zu und dem Einleiten zur Untersuchung von möglicherweise strafrechtlich relevanten Sachverhalten bietet, wird erhöhte Erwartungen an seine Mitarbeiter stellen dürfen, vermeintliche Straftaten zuerst intern zur Aufklärung gelangen zu lassen. Insbesondere wird das der Fall sein, wenn dabei die Anonymität des Mitarbeiters auf dessen Wunsch gewahrt bleiben kann. Der wirtschaftlich-existentielle Schutz des Mitarbeiters wird dann ebenso sichergestellt wie eine geeignete Sachverhaltsaufklärung. Die beispielsweise von einer Innenrevision zu erwartende Sachverhaltsaufklärung wird regelmäßig über das Maß hinausgehen, das von einem einzelnen Mitarbeiter aus dem Unternehmen erwartet werden kann. ${ }^{89}$

Aus strafrechtlicher Sicht verlangt die Möglichkeit der Verdachtsanzeige des Hinweisgebers zwar nicht ebenso ausführliche Ermittlung eines Sachverhaltes wie es die Tätigkeit einer Innenrevision erwarten ließe. Für die Straflosigkeit des Anzeigenerstatters im Fall einer schließlich unbegründeten Anzeige kommt es

8I Hauschka, NJW 2004, 257, 259.

82 Hauschka, ebd.

83 Hauschka, ebd., S. 260; Bannenberg (Fn. 5), S. 2 I 4; LG Bonn, Urteil vom 03.07.200I, NJW 2002, 3260; Stierle, ZIR, 2006, I09, I I 2; OECD-Report On The Application Of The Convention on Combating Bribery of Foreign Public Officials in International Business Transactions And The 1997 Recommendation on Combating Bribery In International Business Transactions No. 78 .

84 Stierle, ZIR 2006, 109 ff.

85 Hauschka, NJW 2004, 260.

86 Bannenberg (Fn. 5), S. 466.

87 BAG NJW 2004, I 547, I 549 .

88 BGH StV i 988, 253, BGH Beschluss v. I4.08.02 (I StR 286/02); BGH Urteil v. 25.06.03 (I StR 469/02).

89 Bannenberg (Fn. 5), S. 25 I; Hauschka, NJW 2004, 257, 260; Stierle, ZIR, 2006; ro9 ff. 
also nicht auf das Vorhandensein einer Innenrevision oder dergleichen mehr von einem Unternehmen ergriffener institutioneller Präventionsmaßnahmen an. Bei der Interessenabwägung im Rahmen des Rücksichtnahmegebotes zur arbeitsbzw. zivilrechtlichen Betrachtung der Anzeigenerstattung wird es dagegen für die Zumutbarkeit der Fortsetzung des Arbeitsverhältnisses für den Arbeitgeber beachtlich sein müssen, ob ein Mitarbeiter einen Sachverhalt, ohne weitere Untersuchungen durch unternehmensinterne Stellen zu ermöglichen, sofort der Staatsanwaltschaft angezeigt hat. Die Einschaltung der vorhandenen Innenrevision oder anderer Stellen des Unternehmens hätte es im Zweifel dem Arbeitgeber erlaubt, nicht nur wirtschaftlichen Schaden wie auch das Risiko eines Imageverlustes abzuwenden oder zu mindern. $9^{\circ}$ Darüber hinaus hätte er auch die Ermittlungsbehörden mit gezielteren Informationen versorgen und dadurch die Strafverfolgung von Mitarbeitern auch im eigenen Interesse effektivieren können.

Neben dem bloßen Vorhandensein institutioneller Präventionsmaßnabmen in der Form bestimmter, vom Unternehmen eingerichteter Stellen, wird es freilich auch auf ihre Erreichbarkeit für die Mitarbeiter im Unternehmen sowie ihre Effektivität ankommen. Das Vorhandensein entsprechender Stellen und die Gelegenheit zur Erreichbarkeit müsste entsprechend innerhalb des Unternehmens und in einer für ein Kündigungsschutzverfahren nachweisbaren, dokumentierten Form gegenüber den Mitarbeitern kommuniziert sein..$^{91}$ Einem potentiellen Whistleblower sollte dabei die Möglichkeit der Anonymität gewährt werden, und zwar entweder durch die Annahme auch von solchen Hinweisen, die von vorn herein anonym sind, wie auch von Hinweisen, bei deren Meldung sich der Hinweisgeber zwar offenbart, jedoch um die Sicherstellung seiner Anonymität im unternehmensinternen Untersuchungsverfahren bittet..$^{2}$ Ebenso wird die Effektivität der Sachverhaltsaufklärung nicht von vorn herein offensichtlich eingeschränkt sein dürfen, etwa aufgrund unternehmensinterner Zuständigkeitsbeschränkungen, denen vorgesehene Anlaufstellen unterliegen. Die Implementierung institutioneller Präventionsmaßnahmen wird das Entdekkungsrisiko begangener Straftaten oder Ordnungswidrigkeiten erhöhen. Die Erwartung der Mitarbeiter, dass Fehlverhalten aufgrund effektiver institutioneller Präventionsmaßnahmen eher aufgedeckt werde, wird zudem zu einer Abnahme tatsächlich begangener Straftaten oder Ordnungswidrigkeiten in einem Unternehmen führen. ${ }^{33}$ Das entspricht dem Interesse des Arbeitgebers an einem Schutz vor Straftaten seiner Mitarbeiter. Aus kriminalpolitischer Sicht muss deshalb an der Implementation unternehmensinterner Aufklärungsinstrumente, die gerade auch die Anonymität von Hinweisgebern schützen, wie ebenso an ihrer Betrachtung durch die arbeitsgerichtliche Rechtsprechung ein hohes Interesse bestehen. Die arbeits- bzw. zivilrechtliche Rechtsprechung sollte institutionelle Präventionsmaßnahmen bei der Frage der Unzumutbarkeit vorrangiger Information des Arbeitgebers über von Mitarbeitern festgestelltes Fehlverhalten berücksichtigen. Die Anforderungen an die Unzumutbarkeit der vorrangigen Information des Arbeitgebers müssten beim Bestehen von institutionellen Präventionsmaßnabmen höhere sein. Im Einzelnen wird von der

90 Stierle, ZIR 2006, I09, i Iо.

9I Vgl. dazu im Ergebnis Bannenberg, Vortrag BKA-Herbsttagung 2002, zu finden am 19.06.2007 unter: http://www.jura.uni-bielefeld.de/Lehrstuehle/Bannenberg/Institute_Projekte/

bka\% 2oherbsttagung\% 20schriftfassung.doc; Stierle, ZIR 2006, I09, I I०.

92 Zum Nutzen der Wahrung der Anonymität von Whistleblowern vgl. Fn. 9; Röhrbein, ZIR 2004, 27I f. 93 Vgl. Fn. 86. 
- Bestehen Stellen, sei es unternehmensintern oder unternehmensextern, an die sich der Whistleblower wenden kann?

- Wird dabei der Persönlichkeitsschutz des Whistleblowers gewahrt und besteht die Möglichkeit des anonymen Hinweises?

- Ist eine effektive Prüfung abgegebener Hinweise durch unternehmensinterne Stellen oder durch vom Arbeitgeber beauftragte Dritte zu erwarten?

- Ist der Mitarbeiter über das Bestehen entsprechender Stellen, die Hinweise über im Betrieb erkanntes Fehlverhalten aufnehmen, ausreichend informiert?

- Ist in der Unternehmenskultur die Anerkennung von Hinweisen auf mögliches Fehlverhalten von Mitarbeitern hinreichend verankert - insbesondere durch entsprechende Kommunikation?

Können die vorstehenden Fragen positiv beantwortet werden, wird man dem Arbeitgeber eine erhöhte Schutzwürdigkeit im Hinblick auf die Einhaltung entsprechender, aus dem Rücksichtnahmegebot abzuleitender Aufklärungs- und Schadensabwendungspflichten durch den Mitarbeiter zugestehen dürfen. Der Arbeitgeber wird in stärkerem Maß darauf vertrauen dürfen, von seinen Mitarbeitern bei festgestelltem Fehlverhalten vorrangig vor den zuständigen Ermittlungsbehörden informiert zu werden. Eine Interessenabwägung zwischen dem Interesse des Mitarbeiters an der Anzeigenerstattung und dem Arbeitgeber am Schutz seines eingerichteten und ausgeübten Gewerbebetriebs wird eher zugunsten des Interesses des Arbeitgebers ausfallen müssen. Denn der Arbeitgeber hat hinreichende Maßnahmen zur Aufklärung und Beendigung von Mitarbeiterfehlverhalten mit strafrechtlichem oder ordnungsrechtswidrigem Hintergrund getroffen.

Die hier angesprochenen institutionellen Präventionsmaßnabmen vermögen insbesondere in größeren Unternehmen und Konzernen das Spannungsfeld zwischen dem kriminalpolitischen Interesse an Straftatenaufklärung und arbeitsrechtlichen Pflichten eines etwaigen Whistleblowers zugunsten des Whistleblowers und damit im Interesse der Straftatenprävention aufzulösen. Zugleich würde im Fall ihrer Einbeziehung in die Rechtsprechung - wie hier erörtert - auch die Rechtssicherheit für beide Seiten, den Arbeitgeber wie auch den Whistleblower, steigen. Einerseits würde eine Präzisierung der Erwartungshaltung des Arbeitgebers gegenüber dem Mitarbeiter möglich, andererseits könnte der zivil- bzw. arbeitsrechtlich definierte Pflichtenkreis eines möglichen Hinweisgebers deutlicher umrissen werden. Das Anliegen, Rechtssicherheit zu erreichen, wird insbesondere auch vor dem Hintergrund einer möglichen Ausdehnung der Korruptionsstrafbarkeit durch das Zweite Korruptionsbekämpfungsgesetz an Bedeutung gewinnen.95 Ein Mehr an Straftatbeständen wird auch zu mehr Ermittlungsverfahren und also auch zu mehr Anzeigen führen. Die Anzeigen werden aber auch in Zukunft zumeist nur von Insidern, den Whistleblowern, erhoben werden können.

Die Zunahme institutioneller Präventionsmaßnabmen bei großen Unternehmen kann zudem Strahlungswirkung auch auf kleinere Unternehmen entfalten. Zulieferer könnten etwa mittelbar von erhöhten Aufklärungspotentialen bei Großunternehmen profitieren, weil dadurch auch die Begehung von Straftaten mit Beteiligung externer Täter erschwert bzw. ihre Aufdeckungsquote erhöht würde. Schließlich könnten so Standards entstehen, die auch von kleinen Unternehmen in angemessener Form umsetzbar wären. 